\title{
A Design for Water Surface Communication System of Ultra Short Base Line Positioning System
}

\author{
FuPeng Qiu, HongJie Wan, XueWei Wang \\ Department of Information Science and Technology \\ Beijing University of Chemical Technology \\ BeiJing, China \\ e-mail: qiufupeng1987@126.com
}

\begin{abstract}
In order to satisfy the requirement of the underwater acoustic positioning system for offshore oil exploration, a design idea for water surface communication system of ultra short base line(USBL) positioning system. The main function of this design is to achieve high-quality communication with underwater acoustic responders. The hardware adopted JZ4760 and the FPGA of altera as the cores. Gold sequence direct sequence spread-spectrum was used on communication system, while modulation was PSK. An appropriate spread-spectrum synchronization mode was presented for the system. MATLAB-based simulation and experiments show that the system is working reliably.
\end{abstract}

Keywords- USBL; spread-spectrum; JZ4760; FPGA

\section{PREFACE}

With the technological developing and the continuing demand for marine resources, underwater acoustic positioning technology ${ }^{[1]}$ has been widely used in civilian applications, such as: exploration of seabed topography, offshore oil development and laying underwater cables. Looking abroad on the technology of underwater acoustic positioning used on offshore exploration, mostly is Sonardyne, Kongsberg Simrad and Nautronix's products ${ }^{[2]}$. These companies represent the advanced level in this field. In China, the product of underwater acoustic positioning is monopolized by these companies, which developing of domestic underwater acoustic positioning system is becoming imperative.

USBL positioning system is the use of acoustic positioning technology to locate underwater targets, tracking and data transmission. USBL positioning system is characterized by the size of the arrays is very small, can be used on a smaller carrier. Emission transducer and several hydrophones can be combined into a whole, known as the sound head. Its size is only a few centimeters to one meter. System array size is small, so the installation more convenient, and equipment costs are relatively low.

This paper focuses on a hardware design for water surface communication system of USBL positioning system. The underwater acoustic channel is a narrow-bandwidth high latency, high noise and severe multipath fading channel ${ }^{3]}$, and so spread-spectrum communication technology was adopted.

\section{USBL SYSTEM INTRODUCTION}

USBL positioning system is mainly composed of four subsystems: the deck unit, the sound head unit, responders and motion reference unit (MRU). The deck unit consists of high-performance computers and dedicated signal processor, complete system control, data processing and display functions; Sound head unit consists of the transmitter and receiver hydrophone array transducer, and the receiving array using four vertical aperture orthogonal cross array; Responder with the duty of the low-power, broadband spread spectrum technology can work underwater for a long time; MRU is the USBL system to obtain underwater responder absolute geographic coordinates of the sensor unit.

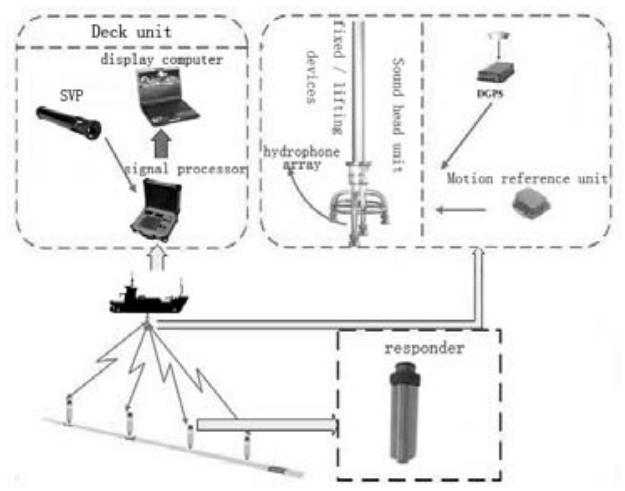

Figure 1. USBL System block diagram

System block diagram shown in Figure 1. Each subsystem is summarized as follows:

- Deck unit: used to display and control and data proce ssing. Including the display and control computer and a dedicated signal processor, including with the sound head, the sound velocity profiler (SVP) and other peripheral interfaces.

- Sound head unit: for positioning the acoustic signal generation, transmit and receive, including the hardware circuit, launching transducer, the receiving hydrophone sensor array and the sound head fixed / lifting devices, including peripheral interface with the MRU and the deck unit. 
- The responder: for the acoustic signal receiving, processing and responses.

- The MRU: for receiving hydrophone array manifold trim, roll and heading angle of the high-precision measurement, the USBL access to essential equipment underwater absolute geographic coordinates.

This paper describes design of the water surface communication system, include the hardware design of sound head unit and the design of spread spectrum communication system.

\section{HARDWARE SYSTEM INTRODUCTION}

\section{A. Hardware system}

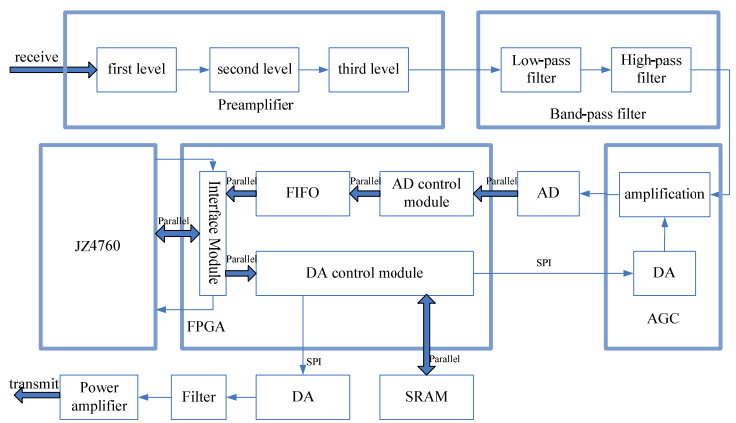

Figure 2. Overall functional block diagram

JZ4760 and FPGA are cores of the water surface communication platform hardware circuit. Main features of the hardware system are:

- By the host computer to control the synchronization sequence and information sequence to generate responder;

- Power amplifier driver transmit transducer sends positioning signals;

- By the receiving water to listen to the array to receive multi-channel responder response signal amplification, filtering, preprocessing the received signal;

- A / D conversion of the received data, and data upload to the host computer;

Sound head hardware design block diagram in Figure 2, in which the host CPU is JZ4760, which is a highperformance 32-bit MIPS-based embedded processor, clocked at $600 \mathrm{MHz}$, can efficiently complete the spread spectrum signal generation, analog front-end of the sound head digital processing and real-time data transfer tasks.

FPGA is EP2C8Q208C8 of altera, with core voltages of $1.2 \mathrm{v}$, the logical resources $8 \mathrm{k}$ available $\mathrm{i} / \mathrm{o}$ port 138 , the storage space 165888bit. Connected to the FPGA through the parallel port and JZ4760, AD, SRAM, SPI and two DA is connected to drive the underlying hardware circuit and realtime data transmission.

First, the sound head master CPU (JZ4760) generates the spread spectrum signal data, to be transmits data to the FPGA through the parallel port. The FPGA will transmit signal data storage in external SRAM. When the FPGA get launch instruction from the CPU, the SRAM data in accordance with a specified frequency to the DA module. DA module to send analog positioning signal, after filtering and power amplification, Final transmit signals by the emission transducers of the sound head.

Responder returns a response signal, the analog part of the hardware system to the receiving four-channel signal through preamplifier, band-pass filter, AGC amplification and ADC sampling. The FPGA controls ADC sampling logic, the data cache in the FIFO inside FPGA. The communication channel of the FPGA and the sound head master CPU is real-time data upload by the internal interface module with Parallel port.

The whole process controlled by the host CPU (JZ4760), and the FPGA drive the each module of bottom. Completion of a high-quality ultrasound spread spectrum communication with the underwater responder.

\section{B. Channels consistency analysis and processing}

USBL positioning algorithm solver the angle base on the phase difference of the signal from the four channels. Channel consistency between the channels directly affecting the positioning accuracy. In accordance with the specific requirements of the algorithm, the phase error in the data collection process must be controlled within $1^{\circ}$. The front of the analog module has been tested in larger phase difference exists, the study found that this is because the device itself there are tiny differences and channel routing slight difference. Late after commissioning, and finally has meet the requirements of phase.

\section{SPREAD-SPECTRUM COMMUNICATION SYSTEM}

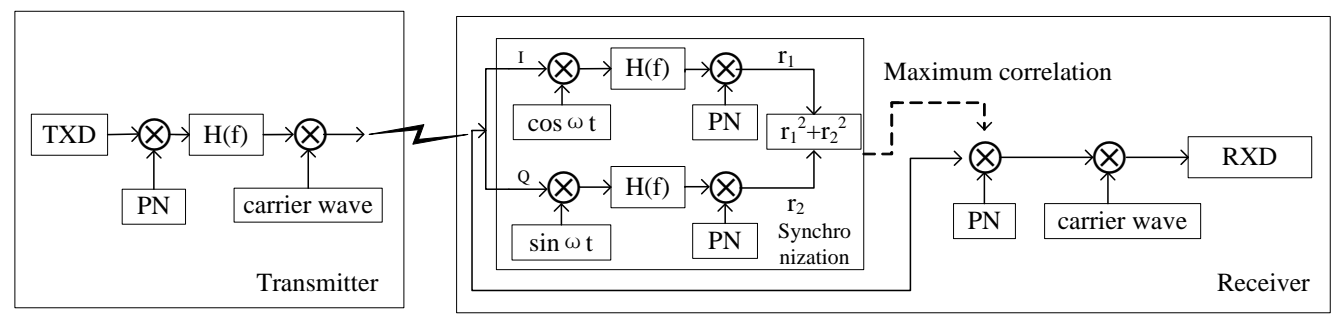

Figure 3. Underwater acoustic communication system block diagram 
The underwater acoustic channel is a time-varying random channel, and the severe multi-path interference and acoustic Doppler shift are main reasons for the high error rate communication. It is an effective method for multiple accesses in acoustic channel which utilizes the orthogonal pseudo-random code to achieve channel's sharing in frequency, time and space [4]. Simultaneously spreadspectrum signal can also effectively reduce the impact of the multi-path signal transmission and improve the ability of interference.

The system requires more than one responder can answer simultaneous, so the communication system used Gold direct sequence spread-spectrum for encoding. Also according to

Design of the PN code selection, 10 bands of Gold code sequence is used in communication system.

The spreading code generated a series of rectangular pulses, when the rectangular pulse through a band-limited channel, the pulse will have a delay spread which leads to the responder's low communication rate, an appropriate spread-spectrum synchronization mode was presented, while modulation was PSK. Spread-spectrum communication system block diagram is shown in Figure 3.

\section{A. Design for transmit signal}

The communication of responder is only to verify whether has received response signal, without any amount of information. As shown in Figure 3, the system sends a PN code as the transmit data, it is through the wave form filter and modulated by carrier wave. In view of the orthogonal

pulse into the adjacent yard intervals and results in intersymbol interference [5]. To solve this problem, a multi-level wave form filter has been adopted.
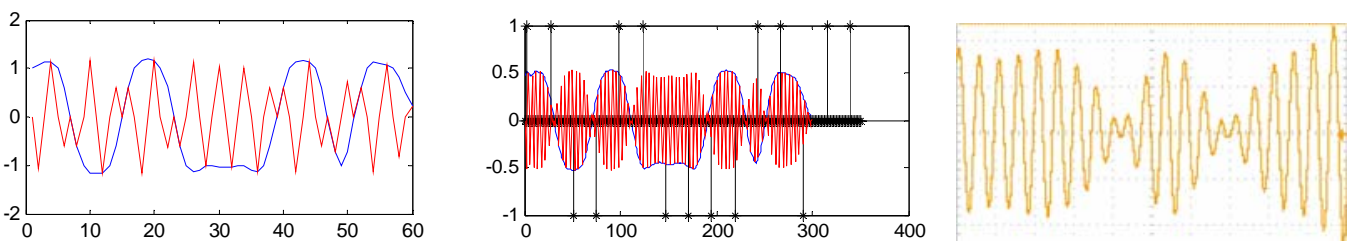

Figure 4. (a)Signal through the shaping filter; (b) Signal through the square root raised cosine filter; (c) Experimental waveform

The signal through a raised cosine filter and a square root raised cosine filter respectively, and liters of sample to meet the requirements of the amplifier circuit. The waveforms based on MATLAB after two filters are shown in Figure 4, the (a) shows the signal through the raised cosine filter, and the (b) means the signal after a square root raised cosine filter. Finally, the signal is modulated by PSK, and transmitted out though the transducer. The (c) is the experimental transmitted signal, in which the transmitted signal designed reliable and met system simulation results

\section{B. Synchronization mode for spread-spectrum communication}

The synchronization is a key step to the spread-spectrum communication, which determines the processing gain and stability of the communication. And it is divided into PN code synchronization and carrier synchronization [6]. Only one single frame data was transferred in communication process, so we proposed a way of spread-spectrum synchronization which could reduce the computational and achieve the precise synchronization.

Shown in Figure 3, the signal received was divided into two paths I and Q, and they were multiplied with the local carriers that phase difference of 90 degrees respectively. Then signals above though wave form filters and completed the down-sampling, later did the correlation operation with responder own PN code respectively which obtained the correlation coefficients $\mathrm{r} 1, \mathrm{r} 2$ and $\mathrm{r} 12+\mathrm{r} 22$. In a series of correlation coefficients to be the greatest moment of the r12+r22 was the time synchronization completed.

Shown in Figure 5, the (a) means the waveform which correlation coefficient $\mathrm{r} 1$ of I road, and the (b) is Q road correlation coefficient $\mathrm{r} 2$, the most important correlation coefficient $r 12+r 22$ is shown in the (c). Seen from the figure, the highest correlation peak is the time synchronization completed, and from this frame we can restore the data of the signal.
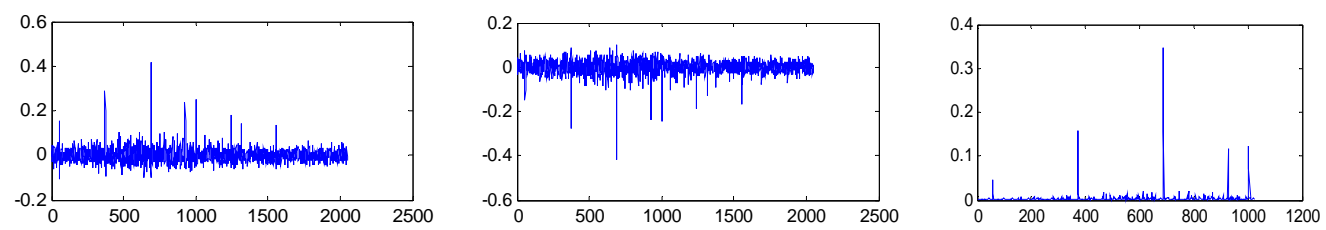

Figure 5. (a)correlation coefficient r1; (b)correlation coefficient r2; (c)correlation coefficient r12+r22 by MATLAB 


\section{CONCLUSION}

This paper presents a hardware design for water surface communication system of ultra short base line positioning system based on the MIPS architecture embedded processor and FPGA, and specific describes the coding processes and synchronization of the spread-spectrum communication system. Meanwhile, the article makes a detailed analysis and testing against the Channel consistency of multi-channel data acquisition. MATLAB-based simulation and experimental verification show that the system has low communication error rate, and supports multi-user communication which reached the desired design specifications. Further applied on the ultra-short baseline underwater acoustic positioning system, it would improve the effectiveness of the work on offshore exploration operations.
[1] ShouJun Li. A Practical Overview and Prospect of Acoustic Positioning Technology.Ocean Technology 2005;(1):130-135.

[2] Fei xiang $\mathrm{Xu}$, Bin Li, Jie Yang. Design of Acoustic Release Responder for Seep Water Deployment.Computer Measurement \& Control 2010;18(5): 1148-1150.

[3] Qin Wang, Lan jun Liu, Xiao tong Zhang. Underwater Acoustic Spread Spectrum Communication System for CDMA Mechanism.Journal of System Simulation 2009;21(24): 7845-7850.

[4] Stojanovic M, Freitag Lee. Multichannel Detection for Wideband Underwater Acoustic CDMA Communications.IEEE Journal of Oceanic Engineering (S0364-9059)2006;31(3): 685-695.

[5] Licheng Yang, Naiping Cheng. Design and realization of shaping filter in spread spectrum communication.Electronic measurement technology 2010; 33(10):44-47.

[6] Changxin Fan, Lina Cao. Communication Theory.6th ed. Beijing: National Defense Industry Press, 2007:404-415. 